\title{
MWA Observations of the EOR1 Field
}

\author{
Bart Pindor ${ }^{1,2}$ \\ ${ }^{1}$ University of Melbourne, School of Physics \\ email: bpindor@unimelb.edu.au \\ ${ }^{2}$ ARC Centre of Excellence for All-sky Astrophysics (CAASTRO)
}

\begin{abstract}
The EOR1 field at $(\mathrm{RA}, \mathrm{DEC})=\left(4 \mathrm{hrs},-30.0^{\circ}\right)$ is one of the main targets of the MWA EOR experiment. It is notable for being in one of the coldest regions of the southern radio sky, as well as for containing the bright radio galaxy Fornax A. We report an early demonstration that the distance of this field from the Galactic Centre may make it a prime field for EOR observations.
\end{abstract}

Keywords. cosmology: observations

\section{Introduction}

The Epoch of Reionization (EOR) was the period of cosmic history during which the first stars and galaxies ionized nearly all of the hydrogen in the intergalactic medium (IGM). The transmission of Ly-A photons from high redshift quasars tells us that this process was essentially complete by $z \sim 6$ (Fan et al. 2006), while the optical depth to Thompson scattering of CMB photons indicates a midpoint of reionization between $7.8<z<8.8$ (Planck Collaboration et al. 2016). The detection of the redshifted $21 \mathrm{~cm}$ radiation from neutral hydrogen in the IGM during this epoch is the goal of a number of ongoing (MWA $†, L O F A R \ddagger$ ) and planned (HERA $\uparrow$, SKA Mellema et al. (2013)) lowfrequency cosmology experiments.

The most difficult problem to be overcome in $21 \mathrm{~cm}$ cosmology are the astrophysical foregrounds which are 4-5 orders of magnitude brighter than the cosmological signal. In this paper, we consider one of the crucial design decisions of all future $21 \mathrm{~cm}$ experiments; how the selection of the observing field affects the foreground contamination.

\section{The MWA EOR Experiment}

The Murchison Widefield Array (MWA Tingay et al. (2013)) is a low-frequency radio telescope situated at the Murchison Radio Observatory in Western Australia; site of the future SKA1-LOW. The MWA EOR experiment is an attempt to statistically detect the fluctuations of the $21 \mathrm{~cm}$ signal at frequencies $\sim 138-198 \mathrm{MHz}$ corresponding to $z \sim 6.1-9.2$. Due to the sky-dominated thermal noise, integrated observations of $1000+$ hrs will be required to achieve a significant detection with the MWA (Beardsley et al. 2013). As a result, the MWA EOR experiment has chosen to concentrate on observations of three main target fields; EOR0 at $(\mathrm{RA}, \mathrm{DEC})=\left(0 \mathrm{hrs},-27.0^{\circ}\right)$, EOR 1 at $\left(4 \mathrm{hrs},-30.0^{\circ}\right)$ and EOR2 at $\left(11.33 \mathrm{hrs},-10.0^{\circ}\right)$. The positions and spacings of these fields are chosen to avoid the brightest regions of the Galaxy while maximizing the available observing time over the course of the year. The deepest MWA EOR limits to date were reported

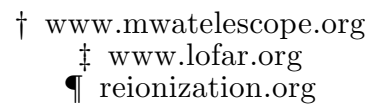




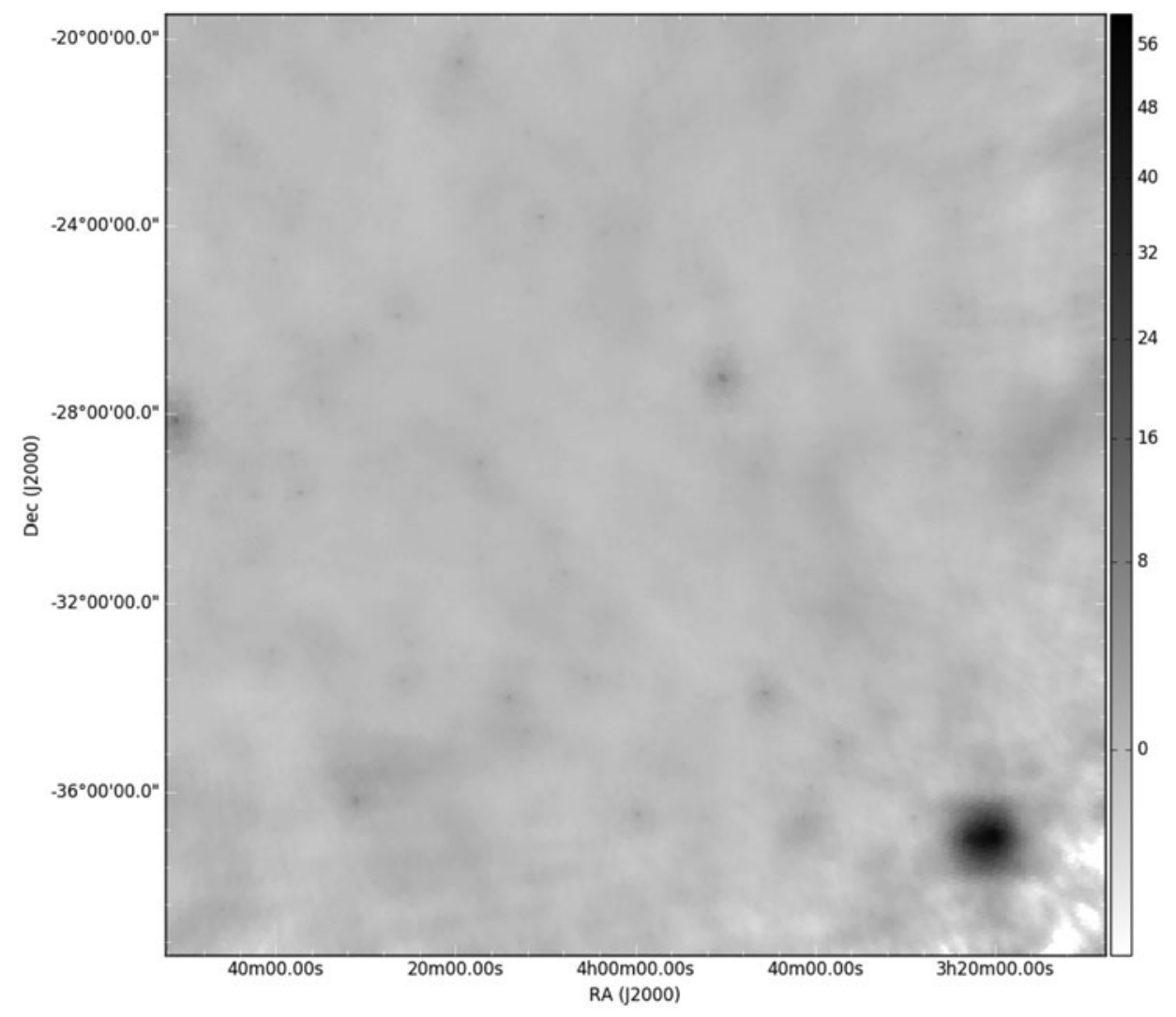

Figure 1. Dirty Image of EOR1 integrated over $\sim 2 \mathrm{~min}$ and $\sim 30 \mathrm{MHz}$

from EOR0 in Beardsley et al. (2016)(B16). Here we report some early comparisons of MWA observations of EOR1 to EOR0; highlighting the relative importance of widefield foregrounds versus bright sources in the main field of view.

\subsection{Processing EOR1}

We process data from the MWA using the Real-Time System (RTS, Mitchell et al. (2008)) to calibrate the visibilities and subtract bright sources. The RTS calibration is a twostep process; first a direction-independent (DI) calibration is found for each $\sim 2$ minutes observation by calibrating to a compound calibrator consisting of the 1000 apparently brightest sources within 20 degrees of the field center for a given observation. Next, the peeling step iteratively solves every $8 \mathrm{~s}$ for the individual ionospheric offsets of the 1000 apparently brightest sources, irrespective of their distance from the field centre, and subtracts them from the DI-calibrated visibilities. These residual calibrated visibilities are then passed to CHIPS (Trott et al. 2016) to form the 2D power spectra. The sky model used by the RTS is derived from a combination of MWA GLEAM (Wayth et al. 2015) and GMRT TGSS ADR1 (Intema et al. 2017) data as described in Procopio et al. (2017). A dirty naturally-weighted image of EOR1 is shown in Fig. 1. Most prominent is the powerful radio galaxy Fornax A at $\left(3.36 \mathrm{hrs},-37.1^{\circ} ; \sim 230 \mathrm{Jy}\right.$ at $\left.170 \mathrm{MHz}\right)$.

The 2D power spectra from 30 minutes of EOR1 high band $(\sim 167-197 \mathrm{MHz})$ are shown in Fig. 2. The left and right panels show the NS and EW instrumental polarizations, respectively. The most prominent features are the horizontal stripes caused by the missing data due to the flagging of heavily aliased fine channels at the edges of the coarse bands, 


\section{:OR1_newpfb_selfCal_peel1000_FQ_Plite10EOR1_newpfb_selfCal_peel1000_EOR1_10}
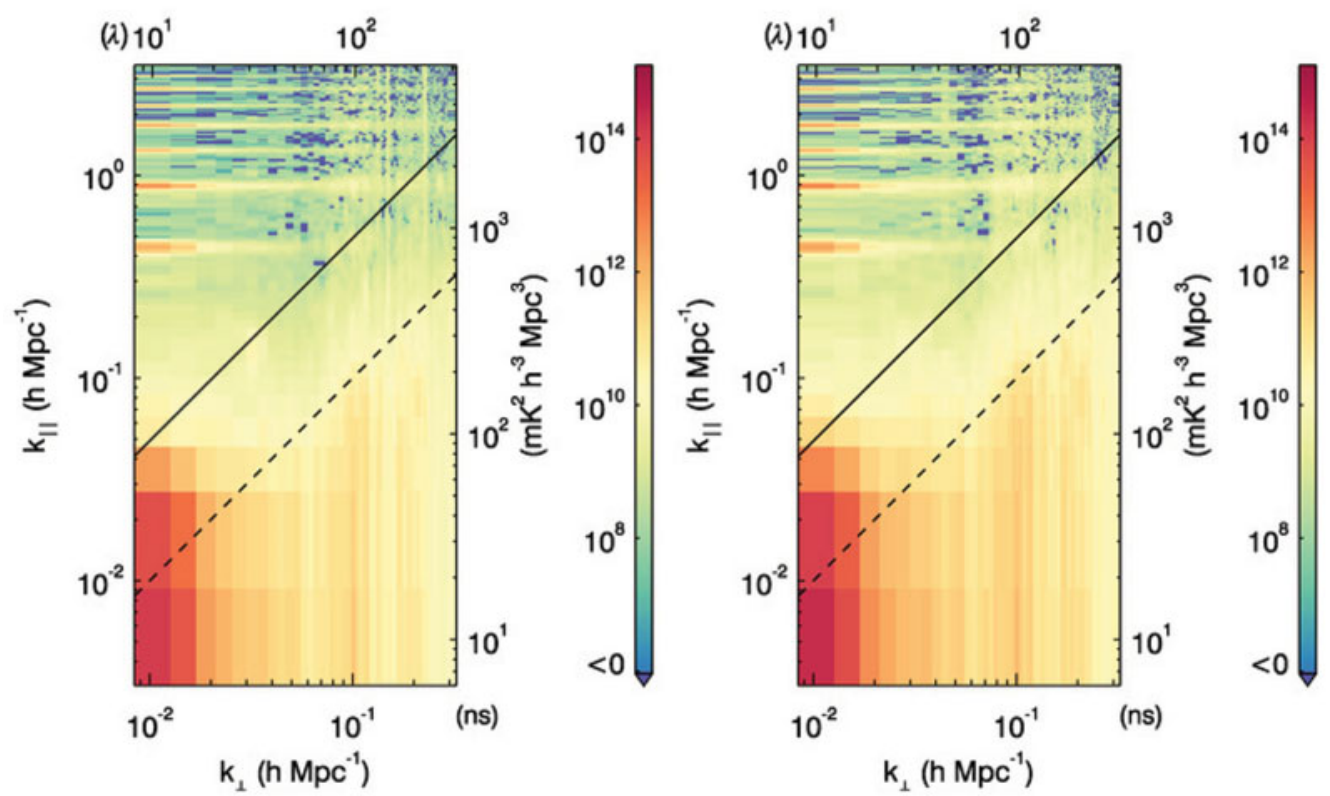

Figure 2. 2D Power Spectra from 30 Minutes of high-band EOR1 observations. NS (left) and EW (right) instrumental polarizations shown.

the concentrated Galactic foregrounds at low $k_{\perp}$ and $k_{\|}$, and the diagonal wedge caused by the chromatic PSF of the instrument.

\subsection{Comparison to EORO}

In contrast to EOR1, the brightest source in EOR0 is GLM235050-2456 ( 10 Jy at $170 \mathrm{MHz}$ ). As a result, it is an ostensibly simpler field to model and could be expected to show lower systematic residuals. However, as shown in B16, the relative proximity of the Galactic Centre $\left(17.75 \mathrm{hrs},-26^{\circ}\right)$ plays a huge role in determining the level of the foreground contamination as the first and second sidelobes of the electronically-formed MWA primary beam pick up very bright Galactic emission. In Fig. 3, we show the difference and ratio of power spectra formed from 30 minutes of zenith data from EOR0 and EOR1.

The left (ratio) panel shows a band of excess relative power in EOR0 running parallel to the black horizon line. This shows that even in this relatively clean EOR0 pointing there is still very clear widefield contamination. Although the small number and regular spacing of the MWA tile dipoles exacerbates the problem of widefield foregrounds, this problem is at some level unavoidable for any interferometer (Thyagarajan et al. 2015). In particular, even the high level of sidelobe suppression offered by the pseudo-random layout of the planned SKA-LOW1 stations will not completely mitigate this effect and it is vital that field selection for the SKA EOR experiments takes all-sky foregrounds into consideration. The right (difference) panel shows that foreground power has been removed throughout the 2D PS space, likely a combination of the generally more detailed sky model as well as the intrinsically colder sky in EOR1. 


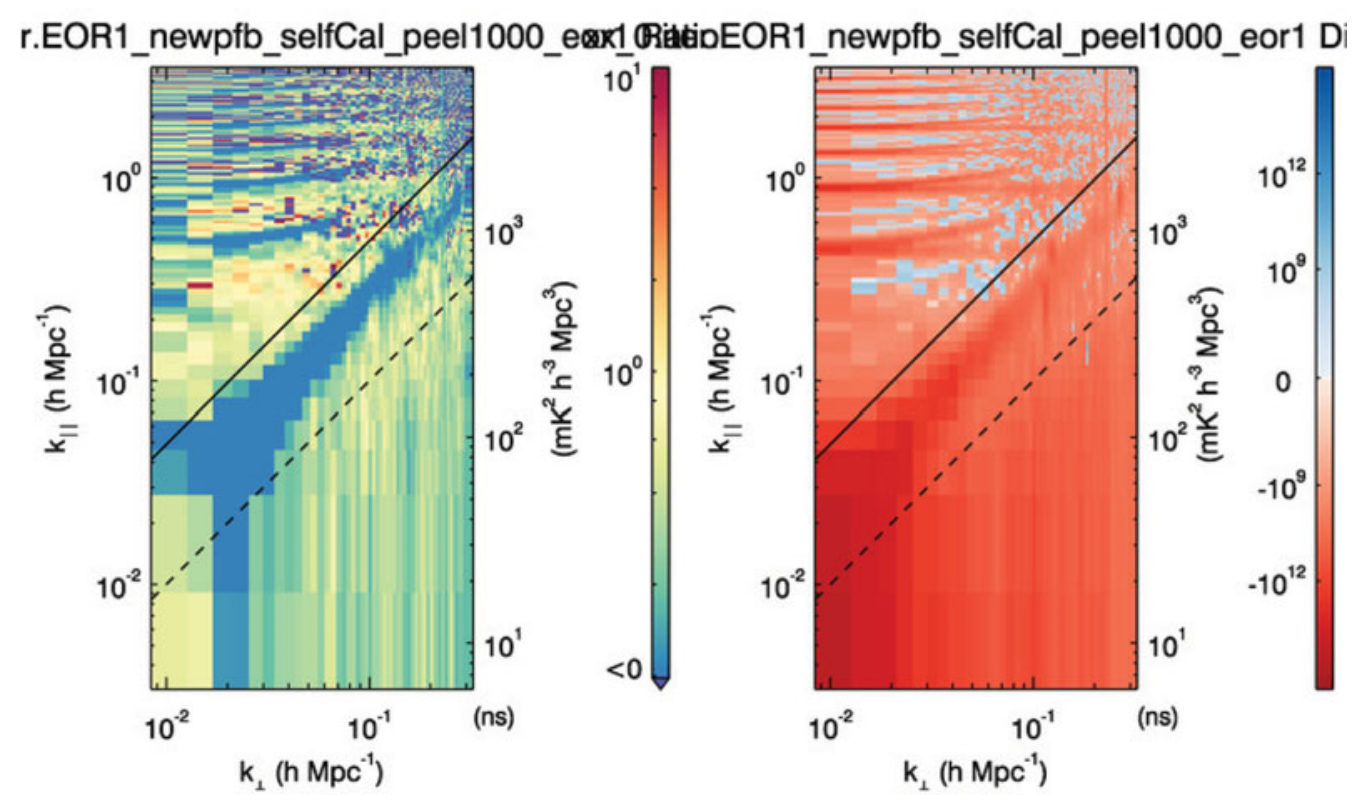

Figure 3. Ratio (left) and difference (right) of $2 \mathrm{D}$ instrumental NS polarization power spectra formed from 30 minutes of high band $(\sim 167-197 \mathrm{MHz})$ for EOR0 and EOR1. The prominent diagonal feature in the left panel indicates that EOR0 has significantly greater widefield foreground contamination (see text).

\section{References}

A. P. Beardsley, B. J. Hazelton, M. F. Morales, et al. MNRAS, 429: L5-L9, Feb. 2013.

A. P. Beardsley, B. J. Hazelton, I. S. Sullivan, et al. ApJ, 833: 102, Dec. 2016.

X. Fan, M. A. Strauss, R. H. Becker, et al. AJ, 132: 117-136, July 2006.

H. T. Intema, P. Jagannathan, K. P. Mooley, \& D. A. Frail. A\& A, 598: A78, Feb. 2017.

G. Mellema, L. V. E. Koopmans, F. A. Abdalla, et al. Experimental Astronomy, 36: 235-318, Aug. 2013.

D. A. Mitchell, L. J. Greenhill, R. B. Wayth, et al. IEEE Journal of Selected Topics in Signal Processing, 2: 707-717, Nov. 2008.

Planck Collaboration A\&S A, 596: A108, Dec. 2016.

P. Procopio, R. B. Wayth, J. Line, et al. PASA, 34: e033, Aug. 2017.

N. Thyagarajan, D. C. Jacobs, J. D. Bowman, et al. ApJ, 804: 14, May 2015.

S. J. Tingay, R. Goeke, J. D. Bowman, et al. PASA, 30: e007, Jan. 2013.

C. M. Trott, B. Pindor, P. Procopio, et al. ApJ, 818: 139, Feb. 2016.

R. B. Wayth, E. Lenc, M. E. Bell, et al. PASA, 32: e025, June 2015. 